\title{
Environmental TEM at Atmospheric Pressure: An Operando View of the Materials at the Nanoscale
}

Ovidiu Ersen ${ }^{1}$, Georgian Melinte ${ }^{1}$, Simona Moldovan ${ }^{1}$, Kassiogé Dembélé ${ }^{1,3}$, Charles Hirlimann ${ }^{1}$, Cuong Pham-Huu ${ }^{2}$, Anne-Sophie Gay ${ }^{3}$ and Sylvie Maury ${ }^{3}$

1. Institut de Physique et Chimie des Matériaux de Strasbourg (IPCMS), UMR 7504 CNRS - Université de Strasbourg, 23 rue du Loess, 67034 Strasbourg Cedex 2, France.

2. Institut de Chimie et Procédés pour l'Énergie, l'Environnement et la Santé (ICPEES), UMR 7515 CNRS - Université de Strasbourg, ECPM, 25 rue Becquerel, 67087 Strasbourg Cedex 2, France.

3. Institut Français de Pétrole - Energies Nouvelles (IFPEN), 69360 Solaize, France.

In the recent years, new approaches based on Transmission Electron Microscopy (TEM) have been developed in an effort to obtain a detailed atomic-scale image of the structural dynamics of materials under conditions mimicking the real working environments. An ever-increasing interest is shown in the development of in-situ gas environments working at low or high pressures and at high temperatures. The closed cells technology within specially designed holders is suitable to mimic the conditions of fullscale reactors and therefore to develop "operando" methodologies for high pressures and temperatures under gas, which are crucial parameters in catalytic reactions. In addition, integrating a residual gas analyzer (RGA) in the gas line outlet of the environmental TEM system allows to directly correlate the structural dynamics of the catalyst and the reaction products. We present herein two "operando" studies carried out in an Atmosphere ${ }^{\mathrm{TM}}$ gas cell (Protochips) [1], which permits atmospheric pressure experiments at temperature up to $1000^{\circ} \mathrm{C}$ together with the analysis of the reaction products. The first is about the mechanisms responsible for the Co catalysts deactivation during hydrocarbons synthesis from syngas in the Fischer-Tropsch (FT) process. The second study approaches the nanostructuration of few layer graphenes (FLG) by Fe nanoparticles (NPs) in a catalytic hydrogenation reaction.

The first study focuses on the stability and reactivity of Co NPs under hydrogen and syngas. The aim was to determine the impact of the structure of these NPs, the 3D architecture of the aggregate and their evolution under gaseous environments at high temperatures on their catalytic activation, reaction yield and deactivation process, the latter induced by the NPs sintering, coking, surface reconstruction or oxidation [2]. In the case of unsupported Co NPs with urchin-like morphology, their exposure to syngas leads to the formation of cobalt carbide species followed by very small changes in their global morphology given the smaller reactivity and reduced atomic diffusion of this phase as compared to the metallic one. When these exotic NPs are deposited on alumina, the atom mobility increases and that leads to their fragmentation during the catalyst activation and to their fast deactivation due to the encapsulation in a coke layer. For an industrial-like catalyst made of Co NPs with an undefined morphology supported on alumina a higher stability was observed when compared to the model systems. However, the environmental study shows the presence of larger NPs after the reactions under hydrogen or syngas which proves the existence of a continuous process of migration and coalescence undergone by the Co NPs. The analysis of the reaction products by the RGA system allowed to correlate the microstructural changes in the catalysts with the catalytic activity and selectivity (Figure 1).

The second in-situ experiment provides a direct insight in the catalytic nanostructuration of FLG under hydrogen. For nanopatterning FLG sheets with well-defined channels, one can activate the catalytic hydrogenation process in which Fe metallic NPs act as mobile nanoreactors able to cut the FLG support 
with a nanometer precision. At the origin of the cutting lies the capacity of NPs to dissolve carbon from the FLG edges and to catalyze the hydrogen-carbon reactions. In a previous study, the 3D TEM analysis provided a deep insight on the impact of NPs morphology and support topography on the catalytic process [3]. We observed that the NPs located at the edges or attached to the steps on the graphene sheets create trenches and tunnels with well-defined orientations, lengths and shapes. However, the $e x$ situ 3D TEM approach offers only a limited perspective on the dynamical behavior of the catalyzed reaction. The real-time motion of the NPs was thus monitored by environmental TEM. We showed that the channeling process implies the continuous carbon dissolution and diffusion from the NP / carbon interface towards the rear and the upper facets of the NP where methane is formed during the hydrogenation of the dissolute $\mathrm{C}$ atoms. A number of aspects relating to the nanoparticle trajectory, such as the changes of the channeling direction (Figure 2) have been also addressed.

On the base of these experiments it is clear that the environmental TEM used in "operando" conditions offers elegant solutions for assessing the materials behavior under real conditions, with particular applications in catalysis. Quantifying the catalyst changes and simultaneously the reaction products provides a complete image of the catalytic reactions and other dynamical processes at the atomic scale.

\section{References:}

[1] Zhang et al, Nature Communication 6 (2015), p. 7778.

[2] Lancelot et al, ACS Catalysis 134 (2014), p. 4510.

[3] Melinte et al, Nature Communication 6 (2015), p. 8071.
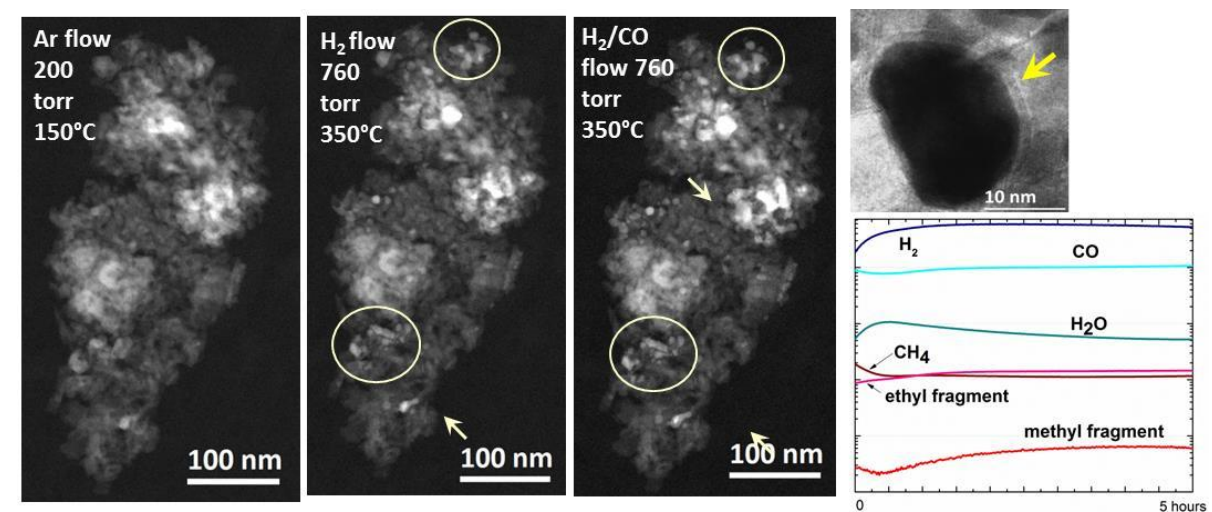

Figure 1. Environmental TEM images recorded on the same Co/alumina aggregate under hydrogen and syngas. On the right: high resolution image on a Co particle showing its encapsulation by graphitic plans (yellow); the real-time analysis of the reaction products under syngas environment by the RGA system.

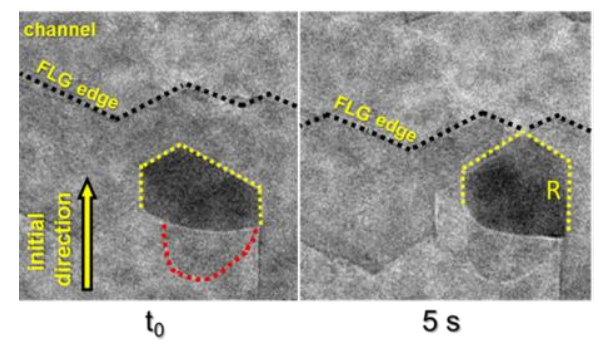

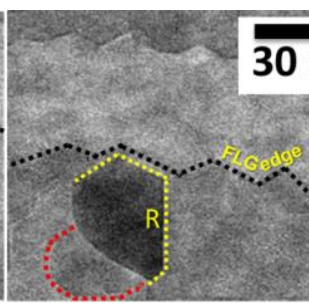

$9 \mathrm{~s}$

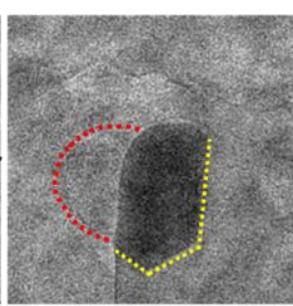

42s

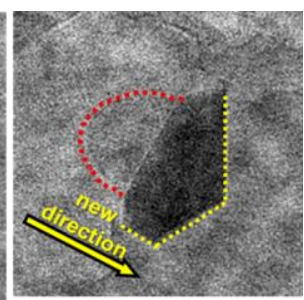

$60 \mathrm{~s}$

Figure 2. Real-time analysis of a particle during the change of the cutting direction when it encounters a topological defect. Yellow lines highlight the front facets and red lines the amorphous carbon tail. 